\title{
Análisis psicométrico del inventario de orientación suicida ISO-19, en adolescentes cordobeses escolarizados
}

\author{
Psychometric analysis of the Inventory of Suicide Orientation ISO-19, \\ in scholarized adolescents
}

\author{
Gerardo Ariel Vecco ${ }^{1}$ *, Pablo Ezequiel Flores-Kanter ${ }^{2,3}$, Leticia Elizabeth Luque ${ }^{1}$ \\ 1 - Facultad de Psicología, Universidad Nacional de Córdoba, Córdoba, Argentina. \\ 2 - Secretaría de Investigación, Universidad Siglo 21, Córdoba, Argentina. \\ 3 - Centro de Bioética, Universidad Católica de Córdoba.
}

Introducción
Método
Resultados
Discusión
Referencias

Recibido: 22/09/2020 Revisado: 05/11/2020 Aceptado: 15/11/2020

\section{Resumen}

Contar con instrumentos válidos y confiables para detectar el riesgo de suicidio es deseable en materia de prevención. En este estudio se explorarán algunas propiedades psicométricas de la versión reducida del Inventario ISO-30 en un grupo de 656 adolescentes escolarizados, de 14-20 años de edad, en Córdoba (Argentina). Para evaluar el ajuste de los datos empíricos al diseño identificado por Galarza, Fernández-Liporace, Castañeiras y Freiberg-Hoffmann (2019) para el ISO-19, se realizaron un análisis factorial confirmatorio (AFC) y un modelamiento exploratorio de ecuaciones estructurales (ESEM). Respecto del AFC, los índices de ajuste del modelo fueron óptimos, levemente inferiores a los obtenidos mediante el modelo ESEM. La cuantificación de la simplicidad factorial identificó 17 ítems por encima del criterio establecido como aceptable. Se concluye que la eliminación de reactivos de la versión original (ISO-30) y los nuevos agrupamientos identificados deben ser considerados para el índice global de orientación suicida que se utiliza en nuestro país.

Palabras clave: adolescencia, suicidio, escala ISO-19, escala ISO-30, propiedades psicométricas

\begin{abstract}
In terms of prevention, it is desirable to have valid and reliable instruments to detect the risk of suicide. Some psychometric properties of the reduced version of ISO-30 are explored, in a group of 656 school-aged adolescents, 14-20 years old, from Cordoba (Argentina). To evaluate the fit of empirical data to the design identified by Galarza, Fernández-Liporace, Castañeiras, and Freiberg-Hoffmann (2019) for ISO-19, a confirmatory factor analysis (CFA) and exploratory structural equation modeling (ESEM) were performed.

Regarding the CFA, the indices for evaluating the model fit were optimal and slightly lower than those obtained using the ESEM model. The quantification of factorial simplicity identified 17 items above the criteria established as acceptable.

The elimination of reagents from the original version (ISO-30) and the new identified groupings should be considered for the global suicide orientation index used in our country.
\end{abstract}

Keywords: adolescence, suicide, ISO 19 scale, ISO 30 scale, psychometric properties

*Correspondencia a: Gerardo A.Vecco. Dirección postal: José Ingenieros 70, dpto. 19, Villa Carlos Paz (CP 5152). Teléfono: +54-3541-516210. E-mail: gerave62@gmail.com

Cómo citar este artículo: Vecco, G. A., Flores-Kanter, P. E., \& Luque, L. E. (2021). Análisis psicométrico del inventario de orientación suicida ISO-19, en adolescentes cordobeses escolarizados. Revista Evaluar, 21(1), 40-52. Recuperado de https://revistas.unc.edu.ar/index.php/revaluar 


\section{Introducción}

La Asociación Argentina de Prevención del Suicidio define al suicidio "como un proceso complejo multideterminado. Una manera de vivir que va construyendo un enigma mortal, por condensación, sobre un marco representacional existente. Un proceso que va más allá del acto, rompiendo la concepción del hecho consumado" (Martínez, 2007, p. 19). Sin negar la evolución continua de los términos y el uso de diferentes expresiones en otros ámbitos del sector, la OMS (2014) entiende por suicidio al acto de matarse deliberadamente y al intento suicida como todo comportamiento que no causa muerte e incluye intoxicación autoinflingida, lesiones y autoagresiones intencionales. El comportamiento suicida incluye la ideación, la planificación, el intento suicida y la comisión del acto. La OMS (2014) aclara que la inclusión de la ideación - como parte del comportamiento- supone un diálogo académico en el que hay diversidad de posiciones no coincidentes.

Desde el punto de vista epidemiológico, el suicidio es considerado un problema grave de salud pública. Por año, alrededor del mundo, se suicidan más de 800.000 personas, lo cual convierte al suicidio en la segunda causa principal de muerte en el grupo etario de entre 15 y 29 años. Asimismo, se estiman 20 intentos por cada suicidio consumado (OMS, 2014). La Dirección de Estadísticas e Información de Salud, del Ministerio de Salud de la República Argentina (DEIS, 2016), informó que entre los años 2014 y 2016 se registraron 1.378 defunciones por suicidio y una tasa trienal de mortalidad adolescente por suicidio de 6.5 por cada 100000 habitantes, entre las que se incluyen las categorías de lesiones autoinfligidas y secuelas de lesiones autoinfligidas de la clasificación CIE-10 de enfermedades y problemas relacionados con la salud. Desde una tasa de $2.5(\mathrm{n}=467)$, en el trienio 1990-1992, la mortalidad de adolescentes por suicidio en nuestro país se elevó a una tasa de 7.2 para el trienio 2011-2013. En 2016, las defunciones adolescentes por suicidio fueron 419 , de las cuales 375 correspondían al grupo de 15 a 19 años de edad y 282 correspondían a varones (DEIS, 2016).

Estos datos indican la relevancia que el tema tiene; sin embargo, la producción empírica sobre el suicidio en Argentina es baja (Flores-Kanter, 2017) y los modelos teóricos predictivos del suicidio evaluados en la región son escasos (FloresKanter, García-Batista, Moretti, \& Medrano, 2019). Por este motivo, resulta imperante contar con instrumentos válidos y confiables para la medición de variables asociadas al riesgo suicida.

En algunos estudios se han indagado los instrumentos disponibles para la medición del riesgo suicida. Rangel-Garzón, Suárez-Beltrán y Escobar-Córdoba (2015), de Colombia efectuaron una revisión de numerosas escalas de riesgo suicida. Su análisis concluye que, si bien algunas escalas cuentan con buenas propiedades psicométricas, la mayoría de los instrumentos son de larga data, con escasos estudios actuales sobre dichas propiedades. Ninguno alcanza elevados índices de sensibilidad y especificidad (lo que implica escasa capacidad predictiva del riesgo suicida) y la complejidad de su modo de aplicación impide su uso en atención primaria.

Runeson et al. (2017) evalúan, mediante un meta-análisis, el riesgo de sesgo y la precisión diagnóstica de instrumentos desarrollados hasta 2014 para la evaluación de riesgo suicida. Concluyen que la mayoría de los instrumentos presentan escasos estudios que evalúen su precisión y, entre los instrumentos analizados, ninguno cumple con los criterios de precisión diagnóstica, a saber: una sensibilidad superior al $80 \%$ y especificidad mayor del 50\%.

Abarca, Gheza, Coda y Elicer (2018) realizaron una revisión bibliográfica sistemática para 
identificar escalas de evaluación de riesgo suicida en adultos usuarios de servicios de atención primaria de salud. Concluyen que ninguna de ellas es lo suficientemente precisa y predictiva para justificar las intervenciones que se realizan con respecto a sus resultados.

De lo expuesto surge la necesidad de estandarizar y revisar constantemente los instrumentos que se utilizan porque de los resultados surgen las propuestas de intervenciones preventivas y posventivas. Es importante tener presente que las pruebas psicométricas se utilizan no solo en investigación, sino también en la toma de decisiones acerca de las posibilidades y oportunidades de vida de las personas (Coolican, 2005).

En Argentina se aplica - tanto con fines investigativos como para la implementación de acciones preventivas - , el Inventario de Orientación Suicida (ISO-30) diseñado por King y Kowalchuk (1994) y adaptado localmente por Casullo (1997). Además, la Subsecretaría de Juventud de la Nación y la Fundación de Prevención del Suicidio lo integran en el Programa de Entrenadores de Vida (Galarza, Fernández-Liporace, Castañeiras, \& Freiberg-Hoffmann, 2019).

Goldston (2000) realizó una revisión de los instrumentos de evaluación de riesgo suicida orientados a niños y adolescentes. Respecto de la escala ISO-30, comunica los índices psicométricos publicados por los autores del diseño original: a) índices de consistencia interna alfa de Cronbach de .92 y .90 para la puntuación total y de .79 y .78 para los ítems críticos, en muestras clínicas y de estudiantes respectivamente; b) fiabilidad test-retest de .80 para la puntuación total y .70 para los ítems críticos, considerando un período de 3-4 días; c) índices de correlación de .64 y .52 para la puntuación total de la escala con el Cuestionario de Ideación Suicida SIQ de Reynolds, y de .55 y .78 con el SIQ-JR de King y Kowalchuk (1994), en muestras clínicas y de estudiantes respectiva- mente. Se informa también que no se encontraron datos publicados respecto a la dimensionalidad o validez predictiva del instrumento, señalando que los métodos de validación se enfocaron solo en la dimensión ideación suicida (Goldston, 2000).

Osman et al. (2005) realizaron un análisis factorial confirmatorio (AFC) sobre el diseño pentafactorial de la escala original (ISO-30; King \& Kowalchuk, 1994), con 202 adolescentes pacientes psiquiátricos hospitalizados, de ambos sexos, de entre 14 y 17 años de edad. Los resultados respaldan el modelo oblicuo original de cinco factores, la consistencia interna del instrumento y sus dimensiones; se informa evidencia de validez discriminante, convergente e incremental. Sin embargo, los autores advierten limitaciones del estudio porque los datos solo respaldan el uso apropiado en adolescentes pacientes psiquiátricos.

Paniagua-Suárez, González-Posada y Rueda-Ramírez (2016) realizaron un estudio psicométrico sobre el ISO-30, en una población de 604 estudiantes adolescentes $(\mathrm{M}=14$ años, $\mathrm{DE}=$ 1.9), 48\% hombres y 52\% mujeres, de Colombia. Se efectuó un AFC, que muestra que los resultados de los índices de bondad de ajuste no están dentro de los parámetros de referencia ideales, pero se considera que la estructura pentafactorial es mejor que cualquier estructura aleatoria alternativa. Se determinó la validez concurrente con la escala RFL-A validada para población colombiana. El índice alfa de Cronbach para la escala completa es de .867. Los autores concluyen que la escala es una herramienta útil para diagnosticar el riesgo de orientación suicida en adolescentes escolarizados de la ciudad de Medellín.

En Argentina se aplica el ISO-30 según la versión adaptada en 1997 por Casullo y, si bien en algunas investigaciones se informa que se utiliza la solución identificada por Fernández-Liporace y Casullo (2006), se sostienen los puntos de corte definidos por la adaptación inicial. Casullo (1997) 
adaptó la escala ISO-30 para ser empleada en adolescentes de Buenos Aires (Argentina). La validez concurrente se obtuvo a partir de una muestra de 30 adolescentes en tratamiento psiquiátrico que presentaban indicadores de potencial suicida (habiendo sido evaluados con técnicas proyectivas); el $80 \%$ obtuvo un puntaje que lo ubicaba como de riesgo suicida alto y el $20 \%$ restante en el nivel moderado. El índice de consistencia interna alfa de Cronbach, para la escala completa, es de .92 en la muestra clínica y de .72 para la muestra de los estudiantes que participaron voluntariamente. El estudio test-retest se efectuó en un lapso de dos semanas entre ambas tomas, con 35 adolescentes estudiantes de una escuela pública de la ciudad de Buenos Aires, y obtuvo una correlación de .86 (Casullo, 1997).

Fernández-Liporace y Casullo (2006) realizaron un estudio psicométrico sobre la versión adaptada del ISO-30 con una muestra de 642 adolescentes de entre 13 y 20 años, escolarizados, de una escuela pública de la ciudad de Buenos Aires. Las autoras analizan la capacidad discriminante de los reactivos y la validación factorial utilizando el método de los componentes principales, con rotación varimax y criterio Kaiser con extracción por contraste de caída. Informan que se eliminan 4 reactivos de la escala original (ítems 10, 19, 28 y 29) y que la solución factorial identificada no responde exactamente a las especificaciones previstas por el diseño, pero que es coherente con la propuesta teórica de base; en virtud de esto, los 5 factores aislados no replican exactamente las dimensiones subyacentes originales y los índices alfa de Cronbach informados son: .88 para el Factor 1 (ideación suicida - ítems 15, 20, 25 y 30), .74 para el Factor 2 (desesperanza- ítems 1, 6, 8, 9, 12, 16, 18, 21, 23 y 24), .67 el Factor 3 (esperan$z a$ - ítems 2, 3, 4, 11, 17, 27), .43 para el Factor 4 (pertenencia interpersonal, que solo incluye dos ítems: 5 y 26) y .50 para el Factor 5 (capacidad de autocontrol y relación con los otros - ítems 7 , 13, 14 y 22). La escala, con 26 ítems, presenta un índice alfa de Cronbach de .87. Se puede apreciar que los factores 4 y 5 presentan índices de consistencia interna por debajo de lo considerado como aceptable por Campo-Arias y Oviedo (2008).

Galarza et al. (2019) realizaron el análisis de las propiedades psicométricas del instrumento en adolescentes y jóvenes universitarios de la ciudad de Mar del Plata (Buenos Aires). Con 821 casos llevaron a cabo un AFE, utilizando análisis paralelo, minimum rank factor analysis robusto para variables ordinales, con rotación promax y normalización Kaiser. Eliminaron 11 ítems (3, 6, 9, $10,13,16,17,19,21,23$ y 28) y obtuvieron una estructura tetrafactorial: Factor 1 denominado ideaciones suicidas (items 15, 20, 25 y 30), Factor 2 como soledad y aislamiento social (ítems 4, 5, 14, 26, 29), Factor 3 como desesperanza (ítems $2,7,11,22,27)$ y Factor 4 como baja autoeficacia (ítems 1, 8, 12, 18, 24). Luego efectuaron un AFC con 297 casos, utilizando como método de estimación ML robusto, comparando el modelo tetrafactorial derivado del procedimiento exploratorio y un modelo pentafactorial, en donde se retienen los mismos 19 ítems, ubicándolos en las siguientes dimensiones: baja autoestima (1, 11, 26), desesperanza $(2,7,12,22$ y 27), incapacidad para afrontar emociones $(8,18)$, soledad y aislamiento social (4, 14, 24 y 29), ideación suicida (5, 15, 20, 25 y 30). Los índices RMSEA son inferiores en ambos modelos a .05, mientras que los índices de ajustes NFI, NNFI, CFI, IFI y MFI superan en ambos modelos .90, presentando el modelo tetrafactorial valores más elevados. Los índices de parsimonia considerados CAIC Independiente y CAIC Modelo indican que el modelo tetrafactorial presenta una mayor parsimonia que el pentafactorial. Los coeficientes alfa ordinales obtenidos para analizar la consistencia interna del modelo tetrafactorial son: $.95, .65, .71$ 
y .69 para los factores ideaciones suicidas, soledad y aislamiento social, desesperanza y baja autoeficacia, respectivamente.

En virtud de lo anterior, el objetivo de la presente investigación fue analizar algunas propiedades psicométricas del Inventario de Orientación Suicida ISO-19 propuesto por Galarza et al. (2019), considerando que no se encontraron antecedentes de análisis factoriales exploratorios o confirmatorios para la provincia de Córdoba (Argentina), teniendo en cuenta las diferencias observadas entre las soluciones factoriales identificadas por Fernández-Liporace y Casullo (2006) y Galarza et al. (2019) y el diseño original (King \& Kowalchuk, 1994), y el hecho de que el instrumento propone medir varios factores asociados a la conducta suicida en lugar de tomar uno o algunos, como ocurre con otras escalas, tales como la CTS (ideas suicidas), PHQ-9 (depresión), el BHS (soledad), entre otras.

\section{Método}

Participantes

Mediante un muestreo no probabilístico accidental (Bologna, 2011) con participación voluntaria, se seleccionaron 656 adolescentes escolarizados, de ambos sexos (52.7\% mujeres y $47.3 \%$ hombres), provenientes de tres instituciones de educación secundaria de la provincia de Córdoba, cuyas edades oscilan entre los 14 y 20 años, con una media de edad de $15.82(\mathrm{DE}=1.342)$ y 15.83 $(\mathrm{DE}=1.348)$. respectivamente.

\section{Instrumento}

El Inventario de Orientación Suicida (ISO30) es una escala que, según King y Kowalchuk (1994), permite evaluar cinco factores asociados al riesgo suicida: baja autoestima, desesperanza, incapacidad para afrontar problemas emocionales, soledad y aislamiento social e ideación suicida. Consta de 30 ítems formulados en forma positiva y negativa (directos e inversos) que se responden mediante una escala Likert de cuatro posiciones: $0=$ Totalmente en desacuerdo, $1=E n$ parte en desacuerdo, $2=$ En parte de acuerdo, 3 = Totalmente de acuerdo. La suma de las respuestas numéricas se expresa en un índice global de la Orientación Suicida que determina la clasificación del riesgo suicida en tres niveles: bajo $\leq 30$; $31 \leq$ moderado $\leq 44$; alto $\geq 45$. Con independencia de la puntuación obtenida, seis reactivos son considerados ítems críticos $(5,10,15,20,25,30)$ ya que aluden directamente a la ideación suicida; la presencia de tres o más ítems respondidos con dos o tres puntos indica la existencia de riesgo elevado.

La ISO-19 (Galarza et al., 2019) es una versión reducida de la ISO-30 que fue adaptada por Casullo (1997) para Argentina. Consta de 19 ítems formulados en forma positiva y negativa (directos e inversos), que se responden mediante una escala Likert de cuatro posiciones: $0=$ Totalmente en desacuerdo, $1=$ En parte en desacuerdo, $2=$ En parte de acuerdo, 3 = Totalmente de acuerdo. Según los autores permite evaluar cuatro factores asociados al riesgo suicida: ideaciones suicidas, soledad y aislamiento social, desesperanza y baja autoeficacia.

El instrumento se administró de forma colectiva a estudiantes de diferentes cursos provenientes de tres instituciones de nivel educativo medio (dos de gestión pública y una de gestión privada), en los horarios normales de clases. La participación fue voluntaria, y se especificó que los datos obtenidos serían tratados de forma confidencial y utilizados exclusivamente con fines investigativos. Previamente, se solicitaron las autorizaciones institucionales y el consentimiento 
informado de padres o tutores; las primeras fueron gestionadas por la dirección de "La Casa del Joven".

\section{Análisis de datos}

Para evaluar el ajuste de los datos empíricos al diseño identificado por Galarza et al. (2019) se realizaron el AFC y el ESEM (Asparouhov \& Muthén, 2009; Ferrando \& Anguiano-Carrasco, 2010; Lloret-Segura, Ferreres-Traver, HernándezBaeza, \& Tomás-Marco, 2014), utilizando los paquetes estadísticos IBM SPSS 23.0 (IBM Corp., 2015), Mplus 6.12 (Muthén \& Muthén, 19982011), y FACTOR 10.8.04 (Lorenzo-Seva \& Ferrando, 2018).

Corresponde aclarar que se complementó el AFC con la aplicación de ESEM, por ser este un método menos restrictivo al no especificar como nulas las cargas secundarias y porque, en ocasiones, las restricciones que impone el AFC podrían no reflejar la complejidad del constructo que se investiga. ESEM presenta una integración de las principales características del AFC, añadiendo la flexibilidad del AFE al estimar en sus análisis el valor de las cargas secundarias. Las investigaciones precedentes que aplicaron ESEM han demostrado la potencia estadística del método en cuanto a los resultados identificados, contribuyendo además en una recuperación rigurosa de las correlaciones interfactoriales informadas e incidiendo en las conclusiones que de ellas se derivan (Trógolo et al., 2020; Dominguez-Lara \& Merino-Soto, 2018; Marsh, Morin, Parker, \& Kaur, 2014; Marsh et al., 2010).

Luego de realizar un análisis de exploración inicial de los datos para identificar casos atípicos (Pérez \& Medrano, 2010), se investigó el grado de asimetría y curtosis considerando como indicadores de alejamiento significativo de la norma- lidad a los valores superiores a tres y diez, respectivamente (Kline, 2005).

Se contempló una matriz de correlaciones policóricas teniendo presente que la escala posee 4 alternativas de respuesta; el índice de Mardia señaló un valor de curtosis superior a 70 cuando se verificó el cumplimiento de los supuestos de la normalidad multivariada, teniendo en cuenta que el índice RMSEA informado fue inferior a .05 (Baglin, 2014; Ferrando \& Lorenzo-Seva, 2014; Lloret-Segura et al., 2014; Freiberg-Hoffmann, Stover, de la Iglesia, \& Fernández-Liporace, 2013; Gadermann, Guhn, \& Zumbo, 2012). La adecuación de la matriz de correlaciones se investigó a partir de la aplicación del test de esfericidad de Bartlett y de la medida de adecuación muestral KMO.

Mediante AFC y ESEM se evaluó el ajuste del modelo utilizando el índice de ajuste comparado (CFI), el índice de Tucker-Lewis (TLI), el error de aproximación cuadrático medio (RMSEA), y el residuo cuadrático medio ponderado (WRMR). Los índices de ajuste obtenidos se analizaron teniendo en cuenta los criterios definidos por $\mathrm{Hu}$ y Bentler (1999), considerando como indicadores de un ajuste aceptable los valores superiores a .90 en los índices CFI y TLI e inferiores a .08 y a 1.00 en RMSEA y WRMR, respectivamente. Se aplicó como método de estimación a los mínimos cuadrados ponderados robustos (WLSMV), cuyo uso resulta recomendado cuando se trabaja con una matriz de correlaciones policóricas y los datos se alejan de la normalidad (Lloret-Segura et al., 2014). Para el modelamiento de ecuaciones estructurales exploratorias (ESEM) se especificó una rotación target.

Respecto de las magnitudes de las cargas principales, se consideró un análisis preliminar desde los criterios que proponen Tabachnick y Fidell (2013), para luego investigar su impacto en la fiabilidad del constructo (Dominguez-Lara, 
Vecco, Flores-Kanter, Luque, Evaluar, 2021, 21(1), 45-52

2018a; Dominguez- Lara, 2018b). Por otra parte, en el modelo ESEM, se evaluó el grado de simplicidad factorial de un ítem mediante el índice ISF (Fleming \& Merino-Soto, 2005; Fleming, 2003), estableciendo como criterio de indicadores aceptables ISF $>.70$ (Dominguez-Lara \& MerinoSoto, 2018; Dominguez-Lara \& Navarro-Loli, 2018).

Para obtener los índices de consistencia interna se calculó el índice omega (Ventura-León \& Caycho-Rodríguez, 2017), considerando aceptables magnitudes que se ubicaran entre $[.70 ; .90]$ (Campo-Arias \& Oviedo, 2008; Ventura-León \& Caycho-Rodríguez, 2017).

\section{Resultados}

Se llevó a cabo un análisis de exploración inicial de los datos con el propósito de detectar la existencia de casos atípicos uni y multivariados. La adecuación de los ítems se determinó a través del análisis de la matriz de correlaciones anti-imagen.

Se calcularon los estadísticos descriptivos (Tabla 1). Los índices de asimetría y curtosis de los ítems analizados presentan magnitudes adecuadas, por debajo de 3 y 10 respectivamente. El índice de Mardia señaló un valor $>70.00$, mientras que RMSEA informó un valor inferior a .05 . Los resultados de aplicar el test de esfericidad de Bartlett $\left(\chi^{2}=2526.2 ; g l=171 ; p<.001 \mathrm{y}\right.$ de la medida de adecuación muestral Kaiser-MeyerOlkin (.839) indican la adecuación de la matriz de correlaciones.

Teniendo en cuenta estos elementos, así como el tamaño de la muestra y que se trabaja con una escala Likert de 4 posiciones, el análisis factorial se realizó a partir de una matriz de correlaciones policóricas.
Tabla 1

Estadísticos descriptivos de los ítems ISO 19.

\begin{tabular}{|c|c|c|c|c|}
\hline & $\mathbf{M}$ & DE & $g_{1}$ & $g_{2}$ \\
\hline Ítem 1 & 1.569 & 1.005 & -.220 & -1.030 \\
\hline Ítem 2 & .529 & .691 & 1.241 & 1.329 \\
\hline Ítem 4 & .697 & .925 & 1.147 & .249 \\
\hline Ítem 5 & .922 & .907 & .597 & -.635 \\
\hline Ítem 7 & 1.384 & 1.028 & .208 & -1.088 \\
\hline Ítem 8 & 2.299 & .941 & -1.165 & .250 \\
\hline Ítem 11 & .742 & .838 & .949 & .185 \\
\hline Ítem 12 & 1.261 & 1.023 & .240 & -1.098 \\
\hline Ítem 14 & .924 & .944 & .763 & -.367 \\
\hline Ítem 15 & .255 & .663 & 2.742 & 6.810 \\
\hline Ítem 18 & 1.738 & .920 & -.423 & -.598 \\
\hline Ítem 20 & .369 & .795 & 2.120 & 3.368 \\
\hline Ítem 22 & 1.063 & .991 & .674 & -.557 \\
\hline Ítem 24 & 1.427 & 1.133 & .034 & -1.400 \\
\hline Ítem 25 & .235 & .658 & 2.927 & 7.762 \\
\hline Ítem 26 & .700 & .943 & 1.089 & -.022 \\
\hline Ítem 27 & .691 & .801 & 1.133 & .924 \\
\hline Ítem 29 & 1.070 & 1.132 & .531 & -1.188 \\
\hline Ítem 30 & .264 & .667 & 2.736 & 6.942 \\
\hline
\end{tabular}

Nota. M: media; DE: desviación estándar $g_{1}$ : asimetría; $g_{2}$ curtosis $p<.05$.

Los índices de ajuste absolutos e incrementales se presentan en la Tabla 2, en tanto los resultados del análisis factorial aplicando AFC y ESEM se informan en la Tabla 3.

En AFC se identificaron índices de ajuste óptimos (CFI $=.966$ y TLI $=.960$; RMSEA [90\%] $=.044[.038-.051])$, ubicándose levemente por encima del valor establecido como criterio, el índice WRMR $=1.092>1.00$. Las cargas factoriales fueron significativas $(p \leq .05)$, y en su mayoría aceptables, con valores que oscilaron entre 
Tabla 2

Índices de ajuste modelo tetrafactorial ESEM y AFC.

\begin{tabular}{lcccccc}
\hline & $\chi^{2}$ & $\boldsymbol{g} \boldsymbol{l}$ & CFI & TLI & WRMR & RMSEA [IC] \\
\hline ESEM & $5733.37^{* * *}$ & 171 & .979 & .965 & .734 & $.042[.034-.050]$ \\
AFC & $5733.37^{* * *}$ & 171 & .966 & .960 & 1.092 & $.044[.038-.051]$ \\
\hline
\end{tabular}

Nota. ${ }^{* * *} p<.001$

.862 y .925 en la dimensión ideaciones suicidas, de .408 a .745 en soledad y aislamiento social, de .457 a .706 en desesperanza, y de .340 a .784 en la dimensión baja autoeficacia. El índice de confiabilidad $\omega$ fue de .939 en la dimensión ideaciones suicidas, de 620 en soledad y aislamiento social, de .696 en desesperanza, y .655 en baja autoeficacia (Tabla 3).

Por otra parte, la salida del AFC informó índices de modificación significativos $\left(\chi^{2}>10\right)$, que implican a 4 de los 5 elementos que agrupa la dimensión soledad y aislamiento social (ítems 4, 14, 26 y 29), y un reactivo de la dimensión baja autoeficacia (ítem 1), y señalan potenciales cargas cruzadas de los ítems 4, 14 y 29 en la dimensión desesperanza, del ítem 26 en las dimensiones $b a$ ja autoeficacia e ideaciones suicidas y del ítem 1 con esta última dimensión (Dominguez-Lara \& Merino-Soto, 2018).

Respecto del modelo ESEM, se obtuvieron índices de ajuste óptimos $(\mathrm{CFI}=.979$; TLI = .965; RMSEA [90\%] = .042 [.034, .050]; WRMR $=.734)$; las cargas factoriales fueron significativas $(p \leq .05)$ y en su mayoría aceptables, con magnitudes que oscilaron entre .749 y .992 en la dimensión ideaciones suicidas, de .322 a .643 en la dimensión soledad y aislamiento social, de .432 a .558 en desesperanza y entre .480 y .556 en baja autoeficacia. En cuanto a la confiabilidad, la dimensión ideaciones suicidas presentó una magnitud de .922 en el coeficiente $\omega$ seleccionado, y en las dimensiones restantes, soledad y aislamiento social, desesperanza y baja autoeficacia se obtuvieron magnitudes de .588, .619 y .622 respectivamente.

La correlación interfactorial promedio en $\operatorname{AFC}\left(\Phi_{\mathrm{AFC}}=|.595|\right)$ fue mayor respecto de la obtenida en $\operatorname{ESEM}\left(\phi_{\mathrm{ESEM}}=|.418|\right)$.

El análisis de simplicidad factorial de las filas (ISF; Kaiser, 1974), a partir del valor de las cargas estandarizadas de los ítems que informa el modelo ESEM, indicó que 15 ítems presentan ISF $>.80$ y otros 2 , ISF $>.70$, señalando en la dimensión soledad y aislamiento social dos reactivos con índices por debajo del valor establecido como criterio para ser considerado aceptable (Dominguez-Lara \& Merino-Soto, 2018): el ítem $4\left(\mathrm{ISF}_{4}=.576\right.$; Confio en que los que se preocupan por mí no me van a abandonar si tengo un fracaso) y el ítem $14\left(\mathrm{ISF}_{4}=.556\right.$; Las personas que son importantes para mi comprenden mis sentimientos; Fleming, 2003; Fleming \& MerinoSoto, 2005).

\section{Discusión}

El suicidio es considerado un problema grave de salud pública a consecuencia del incremento de la tasa de mortalidad relacionada con esta conducta. Según informa la DEIS (2016), en nuestro país la tasa se incrementó trienalmente desde 1990. Dado el carácter relevante que adquieren los instrumentos de evaluación psicológica en el 
Tabla 3

Estructura interna del Inventario de Orientación Suicida ISO 19 (AFC y ESEM).

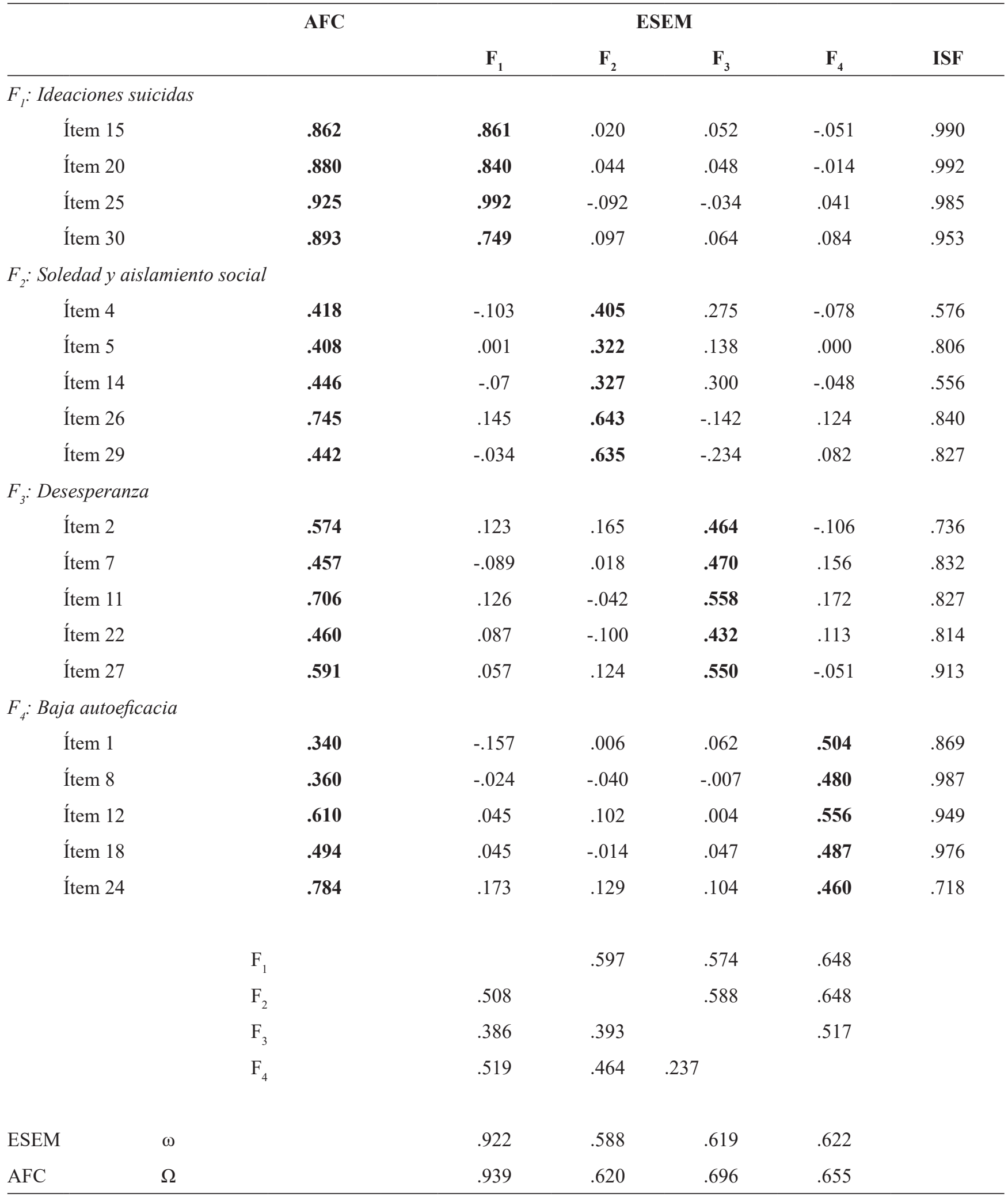

Nota. Sobre la diagonal: correlación interfactorial-método AFC; bajo la diagonal: correlación interfactorial-método ESEM; $\omega$ : coeficiente omega. $p<.05$. 
contexto de la prevención y la detección temprana del sujeto en riesgo suicida, esta investigación tiene por objetivo el estudio de algunas propiedades psicométricas de la versión reducida de 19 ítems de la Escala ISO 30 (Galarza et al., 2019).

El Inventario de Orientación Suicida ISO30 originalmente se organiza en 5 dimensiones: baja autoestima, desesperanza, incapacidad para afrontar emociones, soledad y aislamiento social e ideación suicida. Fernández-Liporace y Casullo (2006) realizan un análisis de las propiedades psicométricas del instrumento, según el cual se eliminan 4 reactivos, y los restantes ítems se reagrupan en 5 componentes que no replican exactamente los agrupamientos del diseño propuesto por King y Kowalchuk (1994). Sin embargo, en Argentina se sigue aplicando la adaptación realizada por Casullo en 1997 o se informa, en algunos casos, que se consideró la solución identificada por Fernández-Liporace y Casullo (2006), pero sosteniendo los puntos de corte definidos para el diseño original de 30 ítems de King y Kowalchuk (1994) adaptado por Casullo (1997).

Galarza et al. (2019) realizan un estudio psicométrico identificando una solución tetrafactorial de 19 ítems que presentan diferencias con la solución factorial identificada por FernándezLiporace y Casullo (2006), y la adaptación realizada por Casullo (1997).

En vista de estas consideraciones, se procedió a realizar los análisis factoriales restrictivos, aplicando AFC y ESEM, con el propósito de evaluar el ajuste de los datos empíricos al modelo tetrafactorial propuesto por Galarza et al. (2019), en una muestra de 656 adolescentes escolarizados de tres instituciones educativas de la provincia de Córdoba.

Como se observó, el ESEM permitió una aproximación rigurosa a la estructura interna hipotetizada; se alcanzaron índices de ajuste óptimos (Hu \& Bentler, 1999). Los ítems en gene- ral estarían representando aceptablemente a las dimensiones que les asignó el diseño. Los parámetros identificados (índices de ajuste, valor de las cargas factoriales, evidencia de diferenciación empírica entre los factores), estarían apoyando en general el diseño propuesto por Galarza et al. (2019).

En cuanto a la cuantificación de la complejidad factorial de las variables observables, estimada a partir de las cargas estandarizadas de los ítems que informa el modelo ESEM, los resultados recolectaron evidencia de su relativa independencia y simplicidad, y señalaron un ajuste empírico adecuado a la estructura factorial que se propone, con excepción de los ítems 4 y 14 que presentan índices de simplicidad factorial por debajo del criterio especificado (ISF > .70; Dominguez-Lara \& Merino-Soto, 2018). Estos elementos aportan evidencias aceptables de que los mismos estarían siendo influidos simultáneamente por más de un factor, lo cual afecta su interpretación e incide en el grado de simplicidad factorial que les supone el diseño teórico (Fleming, 2003; Fleming \& Merino-Soto, 2005; Dominguez-Lara \& MerinoSoto, 2018).

Respecto del AFC, los índices para evaluar el ajuste del modelo fueron óptimos y levemente inferiores a los obtenidos mediante el modelo ESEM, mientras que el índice WRMR indicó un valor de 1.092, ligeramente superior al valor establecido como criterio. Los ítems estarían representando aceptablemente, en general, a sus respectivas dimensiones. Las magnitudes de las correlaciones interfactoriales se incrementaron y eso incide en los resultados y conclusiones que se derivan. Este incremento ha sido investigado, y en su explicación se contempla el efecto de las cargas factoriales secundarias que informa el modelo exploratorio de ecuaciones estructurales (ESEM), y que en AFC se fijaron a 0 (Asparouhov \& Muthén, 2009; Marsh et al., 2014). 
Los índices de modificación que informa la salida del AFC implican a cuatro de los cinco elementos de la dimensión soledad y aislamiento social (ítems 4, 14, 26 y 29) y al ítem 1 del factor baja autoeficacia, en función de lo cual se informan potenciales cargas cruzadas. Además, los ítems 4 y 14 también fueron señalados en la cuantificación de la simplicidad factorial de las filas por presentar índices ISF por debajo del criterio establecido.

La valoración individual de las cargas factoriales es relevante y, al mismo tiempo, objeto de disensos; se inscribe en el debate que se plantea al momento de definir los puntos de corte que se sugieren para retener un ítem y el problema inherente del impacto sobre la fiabilidad del constructo (Dominguez-Lara, 2018a). Los índices de consistencia interna informados en la muestra considerada, que quedan por fuera del rango esperado $[.70 ; .90]$, se vinculan con lo señalado, y aportan evidencia en este sentido (como se observa en la Tabla 3), de modo que sería recomendable que continúen siendo objeto de investigación (Campo-Arias \& Oviedo, 2008; Ventura-León \& Caycho-Rodríguez, 2017).

La modificación en el número de reactivos de la solución tetrafactorial de 19 ítems tendrá implicancias al momento de considerar los puntos de corte que propone el diseño original de King y Kowalchuk (1994), que permiten clasificar al sujeto en tres categorías de riesgo suicida: bajo, moderado o alto $\mathrm{y}$, en particular, la pérdida de dos elementos en la dimensión ideación suicida tendrá incidencias en relación a la otra forma de determinar el nivel de riesgo, ya que el diseño originalmente previsto proponía que tres o más de estos ítems críticos respondidos con valores de dos o tres, indican alto riesgo suicida; por ende, correspondería revisar el indicador total del riesgo, y verificar la capacidad discriminativa del instrumento mediante la definición de curvas ROC, entre adolescentes diagnosticados con y sin intento suicida, con y sin ideaciones suicidas activas.

Como expresa Galarza et al. (2019), la solución tetrafactorial de 19 ítems, con algunas diferencias, sostiene una versión reducida de la escala ISO 30 que debería complementarse con otros estudios que incorporen una muestra más heterogénea en cuanto a variables tales como edad, género, religión, región, condición clínica (a fin de contrastar adolescentes con y sin intento de suicidio previo), propiciando análisis que determinen el nivel de sensibilidad y especificidad del instrumento para establecer puntos de corte; $y$ la recolección de evidencia de validez convergente y discriminante adoptando un criterio externo.

Siguiendo la misma línea de análisis, la eliminación de reactivos y los nuevos agrupamientos identificados en los trabajos presentados por Fernández-Liporace y Casullo (2006) y Galarza et al. (2019), deben ser contemplados cuando se analiza el comportamiento de la distribución de las puntuaciones en una escala y de los factores subyacentes en las muestras consideradas, porque hacen a la fiabilidad y estructura interna de los instrumentos, además de incidir en las conclusiones que de ellos se derivan.

Dada la relevancia del tema, y ubicando el acento en la prevención y detección temprana, se evidencia la importancia que adquiere el análisis de los instrumentos que se utilizan para evaluar el riesgo suicida en adolescentes con el objetivo de mejorar la identificación de sujetos en riesgo suicida y permitirle al profesional psicólogo contar con herramientas más fiables para trabajar con esta problemática.

Por lo tanto, es necesario avanzar en el conocimiento de la problemática del suicidio mediante nuevas investigaciones empíricas que contribuyan tanto en el accionar de políticas en salud pública, como en los tratamientos psicológicos (Flores-Kanter, 2017). 


\section{Referencias}

Abarca, C., Gheza, C., Coda, C., \& Elicer, B. (2018). Revisión de la literatura para identificar escalas estandarizadas de evaluación del riesgo suicida en adultos atendidos en atención primaria de salud. Medwave, 18(05), e7246-e7246. doi: 10.5867/ medwave.2018.05.7246

Asparouhov, T., \& Muthén, B. (2009). Exploratory Structural Equation Modeling. Structural Equation Modeling: A Multidisciplinary Journal, 16(3), 397438. doi: 10.1080/10705510903008204

Baglin, J. (2014). Improving your exploratory factor analysis for ordinal data: A demonstration using FACTOR. Practical Assessment, Research, and Evaluation, 19(5). Recuperado de https://scholarworks.umass. edu/pare

Bologna, E. (2011). Estadística para psicología y educación. Córdoba: Brujas.

Campo-Arias, A., \& Oviedo, H. C. (2008). Propiedades psicométricas de una escala: La consistencia interna. Revista de Salud Pública, 10(5), 831-839. Recuperado de http://www.scielo.unal.edu.co

Casullo, M. M. (1997). Riesgo suicida en estudiantes universitarios. Epidemiología y prevención. Desarrollos en Psiquiatría Argentina, 2(4), 33-38.

Coolican, H. (2005). Métodos de investigación y estadística en psicología. México D. F.: Manual Moderno.

Dirección de Estadísticas e Información en Salud, Ministerio de Salud. (2016). Estadísticas vitales, Información básica, Argentina año 2016. Recuperado de http:// www.deis.msal.gov.ar/index.php/tabulados-2

Dominguez-Lara, S. (2018a). Propuesta de puntos de corte para cargas factoriales: Una perspectiva de fiabilidad de constructo. Enfermería Clínica, 28(6), 401-402. doi: 10.1016/j.enfcli.2018.06.002

Dominguez-Lara, S. (2018b). Fiabilidad y alfa ordinal. Actas Urológicas Españolas, 42(2), 140-141. doi: 10.1016/j.acuro.2017.07.002

Dominguez-Lara, S., \& Navarro-Loli, J. S. (2018). Revisión de metodologías empleadas en los estudios factoria- les de la Escala de Bienestar Psicológico de Ryff (versión en español). Revista Evaluar, 18(2). doi: 10.35670/1667-4545.v18.n2.20800

Dominguez-Lara, S., \& Merino-Soto, C. (2018). Estructura interna del BFI-10P y BFI-15P: Un estudio complementario con enfoque CFA y ESEM. Revista Argentina de Ciencias del Comportamiento, 10(3), 22-34. Recuperado de https://revistas.unc.edu.ar/index.php/racc

Ferrando, P. J., \& Anguiano-Carrasco, C. (2010). El análisis de factorial como técnica de investigación en psicología. Papeles del Psicólogo, 31(1), 18-33. Recuperado de http://www.papelesdelpsicologo.es

Fernández-Liporace, M., \& Casullo, M. M. (2006). Validación factorial de una escala para evaluar riesgo suicida. Revista Iberoamericana de Diagnóstico y Evaluación - e Avaliação Psicológica, 1(21), 9-22. Recuperado de https://www.redalyc.org

Ferrando, P. J., \& Lorenzo-Seva, U. (2014). El análisis factorial exploratorio de los ítems: Algunas consideraciones adicionales. Anales de Psicología, 30(3). doi: 10.6018/analesps.30.3.199991

Fleming, J. S. (2003). Computing measures of simplicity of fit for loadings in factor-analytically derived scales. Behavior Research Methods, Instruments, \& Computers, 35(4), 520-524. doi: 10.3758/ bf03195531

Fleming, J. S., \& Merino-Soto, C. (2005). Medidas de simplicidad y de ajuste factorial: Un enfoque para la evaluación de escalas construidas factorialmente. Revista de Psicología, 23(2), 250-266. doi: 10.18800/psico.200502.002

Flores-Kanter, P. E. (2017). El lugar de la psicología en las investigaciones empíricas del suicidio en Argentina: Un estudio bibliométrico. Interdisciplinaria: Revista de Psicología y Ciencias Afines, 34(1). doi: 10.16888/ interd.2017.34.1.2

Flores-Kanter, P. E., García-Batista, Z. E., Moretti, L. S., \& Medrano, L. A. (2019). Towards an explanatory model of suicidal ideation: The effects of cognitive emotional regulation strategies, affectivity and hope- 
lessness. The Spanish Journal of Psychology, 22. doi: 10.1017/sjp.2019.45

Freiberg-Hoffmann, A., Stover, J. B., de la Iglesia, G., \& Fernández-Liporace, M. (2013). Correlaciones policóricas y tetracóricas en estudios factoriales exploratorios y confirmatorios. Ciencias Psicológicas, 7(2), 151-164. Recuperado de https://revistas.ucu.edu.uy

Gadermann, A. M., Guhn, M., \& Zumbo, B. D. (2012). Estimating ordinal reliability for Likert-type and ordinal item response data: A conceptual, empirical, and practical guide. Practical Assessment, Research \& Evaluation, 17(3). doi: 10.7275/n560-j767

Galarza, A., Fernández-Liporace, M., Castañeiras, C., \& Freiberg-Hoffmann, A. (2019). Análisis psicométrico del Inventario de Orientaciones Suicidas ISO-30 en adolescentes escolarizados (14-18 años) y jóvenes universitarios marplatenses. Revista Iberoamericana de Diagnóstico y Evaluación - e Avaliação Psicológica, 51(2). doi: 10.21865/ridep51.2.10

Goldston, D. (2000). Assessment of suicidal behaviors and risk among children and adolescents. Technical report submitted to NIMH under Contract No. 263-MD-909995. Recuperado de https://vitaalere. com.br/download/assessment-of-suicidal-behaviors-and-risk-among-children-and-adolescents.pdf

Hu, L., \& Bentler, P. M. (1999). Cutoff criteria for fit indexes in covariance structure analysis: Conventional criteria versus new alternatives. Structural Equation Modeling: A Multidisciplinary Journal, 6(1), 1-55. doi: 10.1080/10705519909540118

IBM Corp. (2015). IBM SPSS Statistics for Windows (Version 23.0). [Software de cómputo]. Armonk, NY: IBM Corp.

Kaiser, H. F. (1974). An index of factorial simplicity. Psychometrika, 39(1), 31-36. doi: 10.1007/ bf02291575

King, J. D., \& Kowalchuk, B. (1994). Manual for ISO-30 Adolescent: Inventory of Suicide Orientation-30. Minneapolis: National Computer Systems.

Kline, R. B. (2005). Principles and practice of structural equation modeling ( $2^{\mathrm{a}}$ ed.). New York: Guilford.
Lloret-Segura, S., Ferreres-Traver, A., Hernández-Baeza, A., \& Tomás-Marco, I. (2014). El análisis factorial exploratorio de los ítems: Una guía práctica, revisada y actualizada. Annals of Psychology, 30(3), 11511169. doi: 10.6018/analesps.30.3.199361

Lorenzo-Seva, U., \& Ferrando, P. (2018). FACTOR(Versión 10.8.04). [Software de cómputo]. Recuperado de http://psico.fcep.urv.es/utilitats/factor/index.html

Marsh, H. W., Lüdtke, O., Muthén, B., Asparouhov, T., Morin, A. J. S., Trautwein, U., \& Nagengast, B. (2010). A new look at the big five factor structure through exploratory structural equation modeling. Psychological Assessment, 22(3), 471-491. doi: 10.1037/a0019227

Marsh, H. W., Morin, A. J. S., Parker, P. D., \& Kaur, G. (2014). Exploratory structural equation modeling: An integration of the best features of exploratory and confirmatory factor analysis. Annual Review of Clinical Psychology, 10(1), 85-110. doi: 10.1146/ annurev-clinpsy-032813-153700

Martínez, C. (2007). Introducción a la Suicidología. Buenos Aires: Lugar.

Muthén, L. K., \& Muthén, B. O. (1998-2011). Mplus User's Guide. Sixth edition. Los Angeles, CA: Muthén \& Muthén.

Organización Mundial de la Salud. (2014). Prevención del suicidio: Un imperativo global. Recuperado de https://apps.who.int/iris

Osman, A., Gutierrez, P. M., Barrios, F. X., Bagge, C. L., Kopper, B. A., \& Linden, S. (2005). The Inventory of Suicide Orientation-30: Further validation with adolescent psychiatric inpatients. Journal of Clinical Psychology, 61(4), 481-497. doi: 10.1002/jclp.20086

Paniagua-Suárez, R. E., González-Posada, C. M., \& RuedaRamírez, S. M. (2016). Validación de la versión en español del Inventario de Orientación del Suicidio ISO 30 en estudiantes adolescentes de instituciones educativas en Medellín - Colombia. World Journal of Education, 6(4). doi: 10.5430/wje.v6n4p22

Pérez, E., \& Medrano, L. (2010). Análisis factorial exploratorio: Bases conceptuales y metodológicas. Revista 
Argentina de Ciencias del Comportamiento, 2(1), 58-66. Recuperado de https://revistas.unc.edu.ar

Rangel-Garzón, C. X., Suárez-Beltrán, M. F., \& EscobarCórdoba, F. (2015). Escalas de evaluación de riesgo suicida en atención primaria. Revista de la Facultad de Medicina, 63(4), 707-716. doi: 10.15446/revfacmed.v63.n4.50849

Runeson, B., Odeberg, J., Pettersson, A., Edbom, T., Jildevik-Adamsson, I., \& Waern, M. (2017). Instruments for the assessment of suicide risk: A systematic review evaluating the certainty of the evidence. PLOS ONE, 12(7), e0180292. doi: 10.1371/ journal.pone. 0180292

Tabachnick, B. G., \& Fidell, L. S. (2013). Using Multivariate Statistics (6 ${ }^{\mathrm{a}}$ ed). New York: Allyn and Bacon.

Trógolo, M. A., Tosi, J. D., Poó, F. M., Ledesma, R. D., Medrano, L. A., \& Dominguez-Lara, S. (2020). Factor structure and measurement invariance of the Multidimensional Driving Style Inventory across gender and age: An ESEM approach. Transportation Research Part F: Traffic Psychology and Behaviour, 71, 23-30. doi: 10.1016/j.trf.2020.04.001

Ventura-León, J. L., \& Caycho-Rodríguez, T. (2017). El coeficiente omega: Un método alternativo para la estimación de la confiabilidad. Revista Latinoamericana de Ciencias Sociales, Niñez y Juventud, 15(1), 625-627. Recuperado de https://www.redalyc.org/ pdf/773/77349627039.pdf 